\title{
Análise temática e metodológica da publicação científica em Psicologia no Brasil
}

Thematic and methodological analysis of the scientific publication in Psychology in Brazil Análisis temático y metodológico de la publicación científica en Psicología en Brasil

\author{
Thiago Loreto Garcia da Silva* \\ Carine Capra-Ramos \\ Andreia Rossi Victorazzi \\ Fernanda Carrion da Silva \\ Cristiano Weiss Martins Lima \\ Alice Einloft Brunnet \\ Adolfo Pizzinato**
}

\begin{abstract}
Resumo
A ciência psicológica, por estar situada entre as áreas das ciências humanas e naturais, suscitou diversos debates a respeito de suas epistemologias desde seu início. Tal diversidade implica em uma ampla gama de métodos de pesquisa e áreas de aplicação. Este artigo objetiva, com base em uma pesquisa bibliométrica, traçar um panorama que permita compreender como essa diversidade se dá na produção acadêmica em Psicologia no Brasil. Foi analisado um total de 379 artigos das revistas Qualis A nacionais. Esses foram categorizados conforme suas áreas de estudo, objetivos, delineamentos, métodos de análise dos dados e instrumentos de coleta. Encontrou-se uma predominância de estudos teóricos e estudos aplicados à Psicologia clínica. Porém a multiplicidade de temas abordados reflete o status diverso da Psicologia, ressaltando a impossibilidade de unificação dela sob um único delineamento conceitual.
\end{abstract}

Palavras-chave: Publicação científica, Metodologia, Pesquisa em Psicologia.

Acadêmicos no curso de graduação em Psicologia da Pontifícia Universidade Católica do Rio Grande do Sul e bolsistas do PET Psicologia (Programa de Educação Tutorial Psicologia/MEC/SESu) da PUC RS.

** Doutor em Psicologia da Educação, mestre em Psicologia Social, tutor do PET Psicologia (Programa de Educação Tutorial Psicologia/MEC/SESu) da PUC RS, psicólogo. 


\begin{abstract}
The Psychological Science, by being situated between the areas of human and natural sciences, has raised many debates about their epistemology since its beginning. This diversity implies in a wide range of research methods and application areas. This article aims, from a bibliometric survey, draw a picture that allows one to understand how this diversity is produced in psychology researches in Brazil. Was analyzed 379 evaluated as Qualis A national article. These were categorized according to their areas of study objectives, designs, methods of data analysis and data collection instruments. We found a predominance of theoretical and applied studies to clinical psychology. However, the multiplicity of themes reflects the different status of psychology, emphasizing the impossibility of unifying it under one single conceptual design.
\end{abstract}

Keywords: Scientific publications, Methodology, Research in Psychology.

\title{
Resumen
}

La ciencia psicológica, al situarse entre las áreas de las ciencias humanas y naturales, ha planteado, desde el principio, diversos debates acerca de sus epistemologías. Esta diversidad implica una amplia gama de métodos de investigación y áreas de aplicación. Este artículo tiene como objetivo, basado en una investigación bibliométrica, hacer un dibujo que permita entender cómo esta diversidad aparece en la producción académica en Psicología en Brasil. Se analizaron un total de 379 artículos de las revistas Qualis A nacionales. Estos se clasificaron de acuerdo a su área de estudio, objetivos, diseños, métodos de análisis de datos e instrumentos de recolección de datos. Se encontró un predominio de los estudios teóricos y aplicados a la Psicología clínica. Sin embargo la multiplicidad de temas refleja el status diferente de la Psicología, haciendo hincapié en la imposibilidad de su unificación bajo un único diseño conceptual.

Palabras clave: Publicación científica, Metodología, Investigación en Psicología.

\section{Introdução}

- mbora as ideias a respeito da mente e ou do comportamento que caracterizam boa parte das ideias psicológicas sejam discutidas desde a Antiguidade, foi apenas após a metade do século XIX que a Psicologia teve sua "fundação" como disciplina acadêmica formalmente 
independente, seja da Filosofia, seja da Biologia. O médico alemão Wilhelm Wundt, então um pesquisador na área de Fisiologia e fortemente influenciado pelo impacto do Positivismo no pensamento acadêmico alemão, foi o responsável por dirigir pesquisas a fim de impulsionar o desenvolvimento de uma "nova ciência", dita psicológica. Estimulado por obras como "Elementos de psicofísica", publicado por seu conterrâneo Gustav Fechner, em 1860, Wundt focou-se no objetivo de promover a Psicologia como uma ciência independente, nos moldes do que se definia como ciência no final do século XIX (Schultz \& Schultz, 2006). Juntando e organizando uma diversidade de ideias e pesquisas já lançadas, o pesquisador apresentou "Princípios da Psicologia Fisiológica" (18731874), balizando, enfim, a Psicologia como uma disciplina oficialmente autônoma, com problemas e métodos próprios (Schultz \& Schultz, 2006).

Para Wundt, a Psicologia seria uma mediadora entre as ciências naturais e humanas, tal e como eram entendidas na época (Mariguela, 1995). Dessa forma, Wundt optou por organizar a nova ciência entre diferentes tradições: a experimental (natural) e a social (humana). Como a produção de conhecimento da época baseava-se no paradigma positivista, era enquadrado como científico o estudo que pudesse quantificar e mensurar o fenômeno observado. Wundt deparou, então, com o duplo papel da disciplina: por um lado, o privilégio de poder elaborar experiências laboratoriais e construir leis quantitativas, e, por outro, a realidade psíquica em sua totalidade, considerando que seu objeto é, ao mesmo tempo, pensante e pensado.

Figueiredo (1991) argumenta que essa dualidade estrutural da disciplina faria com que a Psicologia, apesar de ter nascido do bojo de fundamentações de outras ciências, estivesse fadada a nunca encontrar uma única epistemologia que a definisse dentro dos cânones da ciência. Assim, abre-se um campo de divergências e oposições, que refletem as contradiçôes do próprio projeto da Psicologia como uma disciplina única do saber específico. Segundo esse autor, torna-se, assim, inviável uma unificação da Psicologia por meio de uma "paradigmatização" em torno de apenas uma única alternativa. Mariguela (1995) também argumenta nesse sentido, considerando que as diferentes teorias em Psicologia derivam das suas diferentes áreas de aplicação.

Entretanto tal problemática da Psicologia se dá não só com relação à sua fundamentação, mas também se estende para com o seu objeto de estudo. Tratar o "humano" como o simples elemento de estudo 
dessa ciência não é suficiente, ponderam Prado Filho e Martins (2007). É necessário compreender como os sujeitos concretos são delineados a partir da abstração genérica, dizem os autores, além de definir o que é particular no olhar da Psicologia entre as ciências humanas. Essa especialidade pode ser colocada a partir de uma suposta descoberta do "sujeito psicológico", ou mesmo da instalação deste no discurso e na cultura ocidental moderna. Tratar do surgimento de um sujeito no campo da Psicologia, no entanto, "Implica falar da sua colocação como objeto para um discurso científico socialmente autorizado a enunciar verdades a respeito de instâncias psicológicas que compõem este sujeito" (Prado Filho, \& Martins, 2007, p. 14). Os autores argumentam ainda acerca das divergências da Psicologia científica e da diversidade de objetos:

Tomando o nascimento de um conhecimento psicológico de caráter científico no final do século XIX, pode-se observar certa "dança de objetos" nos desenvolvimentos deste campo ao longo do século XX, ligada ao surgimento de várias Psicologias concorrentes entre si, denotando não uma unidade, nem linearidade, e divergência de abordagem dos "fenômenos psicológicos" (Prado Filho \& Martins, 2007, p. 15).

Essas diversidades epistemológicas têm como consequência também diversidades metodológicas, já que é na visão de ciência do pesquisador que o seu método se fundamenta. O método, por sua vez, são as ferramentas, técnicas e procedimentos nos quais o pesquisador buscará subsídio para a sua investigação. $\mathrm{O}$ método é, portanto, uma etapa específica desse processo, enquanto a metodologia é, de forma mais ampla, a estratégia geral usada para a construção do conhecimento (Palmieri \& Martins, 2008).

Por muito tempo, a cientificidade das pesquisas em uma perspectiva metodológica qualitativa foi questionada pela Psicologia "oficial", que buscava eximir-se da discussão sobre a diversidade epistemológica dela e forçar uma definição única de Psicologia, marcada no paradigma positivista. A perspectiva metodológica não pode ser a determinante da cientificidade da produção do conhecimento em Psicologia. Dessa forma, com o avanço das pesquisas qualitativas, é necessário compreender como se caracterizam as diferentes possibilidades de produção do conhecimento em Psicologia, sejam elas mais marcadas por perspectivas de tipo qualitativo ou quantitativo, o impacto de suas diferentes origens e suas consequências no campo de produção do conhecimento na área.

De acordo com Turato (2005), a pesquisa de tradição quantitativa preocuparse-ia com a busca da explicação generalista do comportamento dos objetos e 
estaria baseada no paradigma positivista. Já a pesquisa qualitativa buscaria uma compreensão dinâmica das possibilidades específicas do humano e o paradigma mais influente seria a fenomenologia. Outra diferença importante seria o objeto de estudo das pesquisas, já que o método quantitativo tem como objeto primordial de pesquisa os fatos, enquanto o método qualitativo tem, por outro lado, os fenômenos, suas configurações pela própria definição do pesquisador. Além disso, cabe ressaltar que a pesquisa quantitativa tem como objetivo o estabelecimento matemático das relações causa e efeito. No entanto, a pesquisa qualitativa objetiva a interpretação das relações de significado dos fenômenos, como referido pelas pessoas.

A validade de ambas as perspectivas para a pesquisa em Psicologia, de acordo com Serapioni (2000), não é absoluta. Para ele, os métodos quantitativos são fracos em termos de validade interna, pois nem sempre é possível saber se medem o que pretendem medir. No entanto, são fortes em termos de validade externa, já que os resultados adquiridos tendem a ser generalizáveis. Por outro lado, os métodos qualitativos teriam grande validade interna, pois focalizam as particularidades e as especificidades dos grupos sociais estudados, porém seriam fracos, em validade externa, pois sua possibilidade de generalização é menor.

Apesar de diferentes, e até antagônicos na maior parte das leituras, as duas tradições convivem e se revalidam na produção do conhecimento na Psicologia contemporânea. Segundo Gunther (2006), não se deve escolher por uma pesquisa ou pela outra. É necessário, no entanto, considerar os recursos materiais, temporais e pessoais disponíveis quando se pretende responder a uma pergunta científica e com isso buscar a abordagem metodológica que permita chegar a um resultado que melhor contribua para a compreensão do fenômeno, no mínimo de tempo necessário.

Asdiversidades epistemológicas, metodológicasedemétodos (instrumentais) no campo da pesquisa em Psicologia compõem uma diversidade de saberes e práticas no que se entende por Psicologia. Com base nessa multiplicidade, este artigo pretende compreender como isso se reflete na produção acadêmica brasileira atual e, assim, fomentar um debate sobre como se configura o saber psicológico e científico atual.

\section{Método}

Esta pesquisa consiste em uma revisão de caráter bibliométrico da literatura nacional na área da Psicologia. Os critérios de inclusão dos artigos foram: (a) ser publicado em 2010, (b) estar publicado em 
periódico classificado como Qualis A1 e A2, de acordo com os critérios da área chancelados pela Coordenação de Aperfeiçoamento do Pessoal do Ensino Superior (CAPES); e (c) abordar temas ligados à Psicologia. Nesse sentido, desenvolveu-se um método de classificação que incorporou os critérios objetivos da avaliação nacional de periódicos promovida pela CAPES, com um filtro qualitativo de critério, ou seja, foram considerados para a análise apenas os periódicos nacionais com um predomínio de afinidade epistemológica com a área da Psicologia, de acordo com o explicitado nas missões e objetivos explícitos da publicação.

A classificação dos periódicos é realizada pela CAPES, como missão complementar à avaliação e fomento dos programas de pós-graduação, conforme a base Qualis (Trzesniak, 2006). São várias as características as quais uma revista deve alcançar para atingir o maior grau de pontuação segundo os critérios da CAPES. Um periódico da área da Psicologia, para ser classificado como Qualis A1, deve ter presença nas bases de dados Institute for Scientific Information (ISI) e no PsycInfo; publicação por associação científica com reconhecimento internacional; condição de se tornar referência internacional para a área da Psicologia" (COORDENAÇÃO DE APERFEIÇOAMENTO DE PESSOAL DE NÍVEL SUPERIOR, 2009, p. 2). Já os critérios que avaliam a revista como sendo de Qualis A2, segundo a CAPES (2009, p. 2), são:

Presença no ISI, ou nos três seguintes IBDs: PsycInfo, Scopus e SciELO. OU Presença em dois dos seguintes IBDs: PsycInfo, Scopus e SciELO mais presença em quatro ou mais dos seguintes IBDs: CLASE, LATINDEX, LILACS, PSICODOC, PASCAL, ou REDALYC; atualização (todos os números do ano anterior publicados até março); periodicidade mínima: quadrimestral (revistas generalistas); semestral (revistas de subáreas) (COORDENAÇÃO DE APERFEIÇOAMENTO DE PESSOAL DE NÍVEL SUPERIOR, 2009, p. 2).

Consoante esses critérios de qualificação, as revistas analisadas nesta pesquisa foram: "Psicologia: Reflexão e Crítica", "Revista LatinoAmericana de Psicopatologia Fundamental", "Psicologia: Teoria e Pesquisa", "Estudos de Psicologia (Natal)", "Estudos de Psicologia (PUC Camp)", "Psicologia em Estudo", "Revista Latino-americana de Psicologia", "Agora", "Interamerican Journal of Psychology" e "Psicologia e Sociedade". As revistas "Brazilian Journal of Medical and Biological Research" e "Caderno de Saúde Pública" foram descartadas 
por terem, em seus números, um predomínio absoluto de artigos que não tratavam de temas de interface com a Psicologia, área não contemplada explicitamente em suas missóes e objetivos.

Para levantar e compreender as informaçóes apresentadas nos artigos, foi usada a análise categorial por eixos semânticos, proposta por Clemente-Díaz (1992). Os artigos foram classificados nas seguintes categorias: (a) quanto às revistas em que estes foram publicados; (b) segundo o objetivo do estudo (Gil, 2010); (c) o delineamento do estudo (Gil, 2010); (d) os procedimentos de análise dos resultados; (e) os instrumentos utilizados para a coleta dos dados; (f) o enfoque metodológico dos artigos segundo suas características como estudo quantitativo ou qualitativo; (g) conforme a área do estudo. Essa classificação foi escolhida por se referir a uma relação de áreas de estudo e atuação em Psicologia, segundo a Associação Brasileira de Ensino e Pesquisa em Psicologia (ABEPP) (Leite, 1993). Após a categorização dos estudos, os dados foram analisados descritivamente, com o auxílio do programa SPSS 17.0.

\section{Resultados e discussão}

O total de artigos analisados foi de 379 . Destes, 113 se caracterizaram como estudos quantitativos, 86 qualitativos e 20 artigos apresentam resultados mistos das duas metodologias. Assim, obteve-se um total de 219 pesquisas empíricas, além de 160 estudos de análise teórica. Esses artigos, que não contavam com coletas de dados humanos ou animais de caráter empírico, foram divididos em quatro tipos de estudo: revisões bibliográficas (33), ensaios (111) e resenha de livros (13). Além disso, 3 relatos de experiência foram categorizados como estudos teóricos por não apresentarem dados coletados.

Não foi possível um panorama fidedigno a respeito dos objetivos de todos os estudos analisados. A classificação proposta por Gil (2010) referente aos tipos de objetivos de estudo, propóe que estes sejam entendidos como: exploratórios, descritivos ou explicativos (Gil, 2010). Das 219 pesquisas com coleta de dados empíricos analisadas, apenas 43 se referiam claramente a alguma dessas categorias. Com base nisso, duas hipóteses podem ser inferidas: (a) os artigos, mesmo publicados nas revistas tidas como as melhores da área no País, não definem claramente qual o objetivo a ser alcançado em suas pesquisas; (b) a classificação de Gil (2010) não representa o que se expressa como objetivos de pesquisa. 
Pela análise das revistas usadas em nosso estudo, percebe-se que as revistas nacionais tendem a seguir uma lógica generalista, mesmo que existam algumas revistas que busquem, em suas missões, o predomínio de estudos de uma subárea de pesquisa específica, como pode se observar na tabela 1.

Tabela 1 - Relação das revistas com as áreas sobre as quais os artigos são publicados

\begin{tabular}{lc}
\multicolumn{1}{c}{ Revista } & Áreas dos artigos ${ }^{1}$ \\
\hline Revista Latino-Americana de Psicopatologia Fundamental & 1 \\
Psicologia: Teoria e Pesquisa & 8 \\
Estudos de Psicologia (Natal) & 6 \\
Estudos de Psicologia (PUC Camp) & 7 \\
Psicologia em Estudo & 9 \\
Ágora & 1 \\
Interamerican Journal of Psychology & 11 \\
\hline Psicologia e Sociedade & 10 \\
\hline
\end{tabular}

Mesmo que a maior parte das revistas seja generalista, as poucas que têm um enfoque por subárea podem ter contribuído para o aumento expressivo de artigos dentro das áreas mais frequentes encontradas. Ou seja, as subáreas com mais maturidade, e mais tradição na pesquisa psicológica, além de serem as que predominam nas revistas generalistas, são também aquelas que mais têm revistas de subárea específica. As áreas dos estudos, em sua maioria, são: Psicologia social (127), Psicologia

\footnotetext{
${ }^{1}$ Os números apresentados representam a diversidade das áreas em que os artigos são publicados em cada revista.
} 
clínica (87) e Psicologia do desenvolvimento (42), conforme pode ser observado no gráfico 1 .

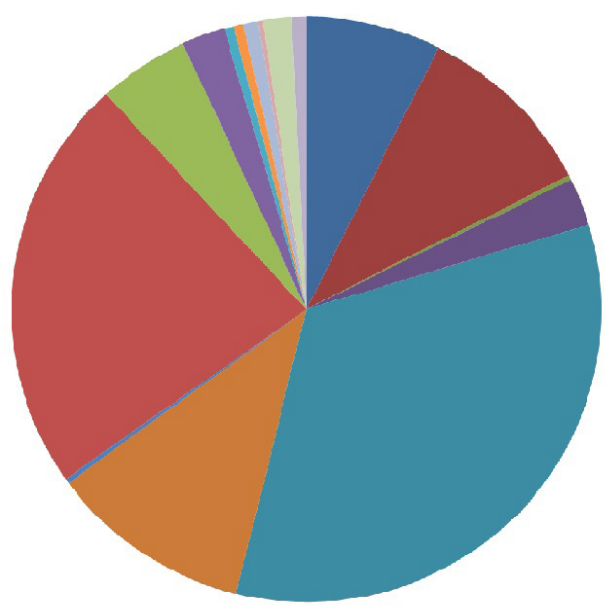

- Fundamendo da Psicologia - História, Teorias, Sistemas 7,4\%

- Metodologia e Avaliação 10,0\%

- Psicologia Experimental H 3\%

- Psicologia fisiológica e neurociência 2,6\%

- Psicologia Social 33,5\%

- Psicologia do Desenvolvimento Humano 11,1\%

- Psicologia da Personalidade 3\%

- Psicologia Clínica 23\%

- Psicologia Educacional e Escolar 5\%

- Psicologia Industrial e da Organização 2,4

- Psicologia Ambiental e Ergonomia 5\%

Laser e Esporte 5\%

- Psicologia Forense 8\%

- Psicologia do Consumidor 3\%

- Formação e Atuação do Psicólogo 1,6\%

- Psicolinguistica 8\%

Gráfico 1 - Proporção de artigos segundo área 
Assim, pode-se fazer uma relação entre esses dados com o que foi encontrado também na análise das outras categorias como, por exemplo, o grande número de artigos teóricos, como pode se observar nas tabelas 2 e 3 .

Tabela 2 - Número de publicações de cada revista de acordo com o método

\begin{tabular}{lcccc}
\hline \multicolumn{1}{c}{ Revistas } & Quantitativa & Qualitativa & Teórico & Misto \\
\hline Ágora & 0 & 3 & 12 & 0 \\
$\begin{array}{l}\text { Estudos de Psicologia } \\
\text { (Natal) }\end{array}$ & 12 & 6 & 10 & 0 \\
$\begin{array}{l}\text { Estudos de Psicologia } \\
\text { (PUC Camp) }\end{array}$ & 15 & 5 & 5 & 0 \\
$\begin{array}{l}\text { Interamerican Journal of } \\
\text { Psychology }\end{array}$ & 23 & 6 & 6 & 5 \\
$\begin{array}{l}\text { Psicologia e Sociedade } \\
\text { Psicologia em Estudo }\end{array}$ & 12 & 16 & 38 & 1 \\
$\begin{array}{l}\text { Psicologia: Reflexão e } \\
\text { Crítica }\end{array}$ & 29 & 22 & 24 & 3 \\
$\begin{array}{l}\text { Psicologia: Teoria e Pes- } \\
\text { quisa }\end{array}$ & 15 & 16 & 10 & 1 \\
$\begin{array}{l}\text { Revista Latino-americana } \\
\text { de Psicopatologia Funda- } \\
\text { mental }\end{array}$ & 1 & 66 & 160 & 20 \\
\begin{tabular}{l} 
Total \\
\hline
\end{tabular} & 113 & 69 & 0 \\
\hline
\end{tabular}

Tabela 3 - Análise dos métodos conforme área de pesquisa

\begin{tabular}{lcccc}
\hline \multicolumn{1}{c}{ Áreas } & Quantitativa & Qualitativa & Teórico & Misto \\
\hline $\begin{array}{l}\text { Fundamento da Psicologia } \\
\text { - história, teorias, sistemas }\end{array}$ & - & 3 & 24 & 1 \\
$\begin{array}{l}\text { Metodologia e avaliação } \\
\begin{array}{l}\text { Psicologia experimental } \\
\text { humana }\end{array}\end{array}$ & 30 & 3 & 4 & 1 \\
$\begin{array}{l}\text { Psicologia fisiológica e } \\
\text { neurociência }\end{array}$ & 4 & - & 1 & - \\
$\begin{array}{l}\text { Psicologia social } \\
\text { Psicologia do desenvolvi- } \\
\text { mento humano }\end{array}$ & 21 & - & 4 & 2 \\
\end{tabular}




\begin{tabular}{lcccc}
\hline \multicolumn{1}{c}{ Áreas } & Quantitativa & Qualitativa & Teórico & Misto \\
\hline $\begin{array}{l}\text { Psicologia da personali- } \\
\text { dade }\end{array}$ & - & - & - & 1 \\
$\begin{array}{l}\text { Psicologia clínica } \\
\text { Psicologia educacional e }\end{array}$ & 22 & 17 & 47 & 1 \\
escolar & 10 & 4 & 5 & - \\
$\begin{array}{l}\text { Psicologia industrial e da } \\
\text { organização }\end{array}$ & 4 & 2 & 2 & 1 \\
$\begin{array}{l}\text { Psicologia ambiental e } \\
\text { ergonomia } \\
\text { Lazer e esporte }\end{array}$ & 1 & - & - & 1 \\
$\begin{array}{l}\text { Psicologia forense } \\
\text { Psicologia do consumidor }\end{array}$ & 1 & - & 1 & 1 \\
$\begin{array}{l}\text { Formação e Atuação do } \\
\text { Psicólogo }\end{array}$ & 1 & 2 & 1 & - \\
Psicolinguística & 1 & 2 & - & - \\
Total & 113 & 86 & 160 & 20 \\
\hline
\end{tabular}

Como se pode observar na tabela 2 , há um predomínio de artigos de natureza quantitativa $(29,8 \%)$ e de estudos teóricos $(42,2 \%)$ nas pesquisas publicadas em revistas no estrato A do sistema Qualis de categorização nacional. Algumas revistas têm uma caracterização bastante específica a respeito do enfoque metodológico dos artigos publicados. Podem-se citar como exemplo as revistas "Ágora" e "Revista Latino-americana de Psicopatologia Fundamental", que predominantemente publicam artigos teóricos.

Uma análise dos perfis metodológicos dos artigos também pôde ser realizada, considerando tanto o delineamento do estudo quanto o procedimento usado para análise dos dados. Essa relação é apresentada na tabela 4.

Tabela 4 - Procedimento usado para análise de dados relacionados com o delineamento de pesquisa

\begin{tabular}{lccccc}
\hline & $\begin{array}{c}\text { Análises } \\
\text { estatísticas }\end{array}$ & $\begin{array}{c}\text { Análises de } \\
\text { conteúdo }\end{array}$ & $\begin{array}{c}\text { Análises de } \\
\text { discurso }\end{array}$ & $\begin{array}{c}\text { Análise de } \\
\text { documentos }\end{array}$ & Outros \\
\hline Transversal & 24 & - & - & - & 2 \\
Validação & 18 & - & 1 & - & - \\
Ext post facto & 3 & - & - & - & - \\
Experimental & 19 & - & - & - & - \\
Ensaio clínico & 7 & - & - & - & - \\
Não especificado & 3 & 1 & 1 & - & 4
\end{tabular}




\begin{tabular}{|c|c|c|c|c|c|}
\hline & $\begin{array}{c}\text { Análises } \\
\text { estatísticas }\end{array}$ & $\begin{array}{l}\text { Análises de } \\
\text { conteúdo }\end{array}$ & $\begin{array}{l}\text { Análises de } \\
\text { discurso }\end{array}$ & $\begin{array}{c}\text { Análise de } \\
\text { documentos }\end{array}$ & Outros \\
\hline Longitudinal & 5 & 2 & - & - & 1 \\
\hline Transcultural & 1 & - & - & - & - \\
\hline Pesquisa-ação & - & 2 & - & - & 2 \\
\hline $\begin{array}{l}\text { Relato de expe- } \\
\text { riência }\end{array}$ & - & 1 & - & - & 0 \\
\hline Estudo de caso & 1 & 3 & 1 & - & 19 \\
\hline Estudo etnográfico & - & - & - & - & 3 \\
\hline Correlacional & 31 & 1 & - & - & 8 \\
\hline Levantamento & - & 20 & 11 & 10 & 14 \\
\hline Total & 112 & 30 & 14 & 10 & 53 \\
\hline
\end{tabular}

Observa-se que, nas pesquisas, há uma tendência de aproximação entre a análise de dados utilizada e o delineamento da pesquisa. Esse padrão segue uma afinidade epistemológica evidente, como, por exemplo, estudos experimentais terem, de forma unânime, análises estatísticas como meio de avaliação de resultados. Porém essa relação não deve ser tomada como regra, pois existem estudos onde variam o tipo de análise independentemente do seu delineamento. Nesse caso, têm-se como exemplo as pesquisas longitudinais que podem ser tanto quantitativas (análises estatísticas) quanto qualitativas (análise de conteúdo), por exemplo.

Há também uma tendência de afinidade entre o uso de instrumentos de coleta de dados de forma coerente com o tipo de pesquisa realizada nas diferentes áreas. Essa relação pode ser observada na tabela 5.

Tabela 5 - Instrumentos de coleta de dados dos estudos em relação a sua área de pesquisa

\begin{tabular}{lccccccccccc}
\hline \multicolumn{1}{c}{ Área } & $\begin{array}{c}\text { Entrev- } \\
\text { istas }\end{array}$ & $\begin{array}{c}\text { Instru- } \\
\text { mentos } \\
\text { psicomé- } \\
\text { tricos }\end{array}$ & $\begin{array}{c}\text { Ques- } \\
\text { tion- } \\
\text { ários }\end{array}$ & $\begin{array}{c}\text { Gru- } \\
\text { pos } \\
\text { focais }\end{array}$ & $\begin{array}{c}\text { Docu- } \\
\text { men- } \\
\text { tos }\end{array}$ & $\begin{array}{c}\text { Não } \\
\text { espe- } \\
\text { cifica- } \\
\text { dos }\end{array}$ & $\begin{array}{c}\text { Não } \\
\text { têm }\end{array}$ & $\begin{array}{c}\text { Ou- } \\
\text { tros }\end{array}$ & $\begin{array}{c}\text { Mis- } \\
\text { to }\end{array}$ & $\begin{array}{c}\text { Tarefas } \\
\text { nado } \\
\text { padro- }\end{array}$ & Total \\
nizadas & \\
\hline $\begin{array}{l}\text { Metodo- } \\
\text { logia e } \\
\text { avaliação }\end{array}$ & 2 & 26 & - & - & 1 & 2 & 4 & 1 & 1 & 1 & 38 \\
\hline $\begin{array}{l}\text { Psicolo- } \\
\text { gia social }\end{array}$ & 26 & 10 & 7 & 5 & 8 & 4 & 59 & 6 & 1 & 1 & 127 \\
\hline
\end{tabular}




\begin{tabular}{|c|c|c|c|c|c|c|c|c|c|c|c|}
\hline Área & $\begin{array}{l}\text { Entrev- } \\
\text { istas }\end{array}$ & $\begin{array}{l}\text { Instru- } \\
\text { mentos } \\
\text { psicomé- } \\
\text { tricos }\end{array}$ & $\begin{array}{l}\text { Ques- } \\
\text { tion- } \\
\text { ários }\end{array}$ & $\begin{array}{l}\text { Gru- } \\
\text { pos } \\
\text { focais }\end{array}$ & $\begin{array}{l}\text { Docu- } \\
\text { men- } \\
\text { tos }\end{array}$ & $\begin{array}{l}\text { Não } \\
\text { espe- } \\
\text { cifica- } \\
\text { dos }\end{array}$ & $\begin{array}{l}\text { Não } \\
\text { têm }\end{array}$ & $\begin{array}{l}\text { Ou- } \\
\text { tros }\end{array}$ & $\begin{array}{l}\text { Mis- } \\
\text { to }\end{array}$ & $\begin{array}{c}\text { Tarefas } \\
\text { não } \\
\text { padro- } \\
\text { nizadas }\end{array}$ & Total \\
\hline $\begin{array}{l}\text { Psicolo- } \\
\text { gia do } \\
\text { desenvol- } \\
\text { vimento } \\
\text { humano }\end{array}$ & 6 & 6 & 5 & 1 & - & 1 & 9 & 4 & 9 & 1 & 42 \\
\hline $\begin{array}{l}\text { Psico- } \\
\text { logia } \\
\text { clínica }\end{array}$ & 4 & 12 & 5 & - & - & 11 & 47 & 2 & 6 & - & 87 \\
\hline $\begin{array}{l}\text { Funda- } \\
\text { mentos } \\
\text { da Psico- } \\
\text { logia - } \\
\text { história, } \\
\text { teorias, } \\
\text { sistemas }\end{array}$ & - & - & - & - & 1 & 2 & 24 & 1 & - & - & 28 \\
\hline $\begin{array}{l}\text { Psicolo- } \\
\text { gia edu- } \\
\text { cacional } \\
\text { e escolar }\end{array}$ & 2 & 3 & 3 & - & 1 & - & 5 & 1 & 2 & 2 & 19 \\
\hline $\begin{array}{l}\text { Psicolo- } \\
\text { gia fisio- } \\
\text { lógica e } \\
\text { neuro- } \\
\text { ciência }\end{array}$ & - & 2 & - & - & - & - & 4 & 2 & - & 2 & 10 \\
\hline $\begin{array}{l}\text { Psicolo- } \\
\text { gia indus- } \\
\text { trial e da } \\
\text { organiza- } \\
\text { ção }\end{array}$ & 2 & 4 & - & - & - & - & 2 & 1 & - & - & 9 \\
\hline $\begin{array}{l}\text { Forma- } \\
\text { ção e } \\
\text { atuação } \\
\text { do psicó- } \\
\text { logo }\end{array}$ & 1 & - & - & - & - & 1 & 3 & - & - & 1 & 6 \\
\hline Outros & 2 & 1 & 3 & - & 1 & - & 3 & 2 & - & 1 & 13 \\
\hline Total & 45 & 64 & 23 & 6 & 12 & 21 & 160 & 20 & 19 & 9 & 379 \\
\hline
\end{tabular}

Subáreas que publicam estudos majoritariamente qualitativos, por exemplo, apresentam um predomínio do uso de entrevistas como ferramenta metodológica, como a Psicologia social, por exemplo. Também, pode-se destacar a área de metodologia e avaliação psicológica, que, por sua vez, tem sua produção calcada majoritariamente no uso de instrumentos psicométricos 
como forma de coleta de dados. Ainda considerando tal questão, deparase a configuração das pesquisas de algumas subáreas com maior rigidez instrumental, não apresentando nenhuma pesquisa utilizando determinados instrumentos. Por exemplo, a subárea da Psicologia fisiológica e neurociências não apresentou nenhum estudo utilizando entrevistas, grupos ou documentos, limitando-se a instrumentos de mensuração de maneira congruente com a lógica das ciências naturais, que orienta tal subárea de pesquisa.

\section{Considerações finais}

Com base nos dados obtidos em nossa pesquisa, podemos inferir que a publicação científica brasileira atual em Psicologia reflete a multiplicidade dos temas existentes nesse campo do saber e os impasses da diversidade metodológica de suas origens. Tal panorâmica, presente nas publicações tidas como as de melhor qualidade nacional, expressa uma realidade na qual parte das discussões iniciadas por Wundt ainda se mantém ativa. Essa diversidade metodológica e epistemológica persiste e aparentemente se configura de forma não integrada, existindo pouca comunicação entre as diferentes tradições que organizam a pesquisa em Psicologia (como pode ilustrar o pequeno número de produções que se configura metodologicamente de forma híbrida ou mista). Essa situação corrobora a ideia de Figueiredo (1991) a respeito das dificuldades da Psicologia em se estabelecer como ciência única ou unificada. Devido e essa pulverização de conteúdos e métodos de estudo nas pesquisas analisadas, parece ser improvável qualquer projeto de unificação da ciência psicológica que não resvale a um profundo reducionismo.

Apesar de não ter sido o foco de nosso estudo, no processo de desenvolvimento desta pesquisa, foi frequente a dificuldade de análise dos artigos devido à não organização do texto de uma maneira clara e compreensível. Um exemplo disso foi a dificuldade de categorização e análise dos objetivos dos artigos. Pode-se pensar que tal carência de informação deve-se à falta de exigência clara dessa discriminação nos projetos editoriais de muitas revistas altamente qualificadas na área. A explicitação de delineamento poderia ser uma de forma a atender a modelos coerentes de organização do texto científico em Psicologia, tal como ocorre em outras áreas, independentemente da matriz epistemológica eleita (Trzesniak \& Koller, 2009).

No que se refere ao rigor metodológico utilizado nas publicações, este estudo, com base na constatação de tal multiplicidade de procedimentos de pesquisa, pode instrumentalizar o debate a respeito da validade e do uso dado à diversidade de métodos científicos (ou não) em que a pesquisa nacional em 
Psicologia se apoia. Tendo em vista que os métodos quantitativos e qualitativos, por exemplo, tem diferentes fraquezas e fortalezas, sugere-se que este debate prossiga no sentido de encontrar parâmetros de rigor de execução desses procedimentos, nas diferentes tradiçôes, em vez da já obsoleta e redundante discussão a respeito de qual tradição epistemológica é superior, assim como os métodos delas advindos.

Considerando que as publicações em Psicologia atualmente são predominantemente fruto de pesquisas geradas em programas de pósgraduação (diretamente influenciados, se não governados pelos órgãos de fomento a pesquisa), tais pontos levantados perpassam por questóes políticas, tanto em termos de política científica, como na política de ocupação dos espaços com poder de influência nas referidas agências (Waters, 2006; Castro, 2010). Segundo Rocha-e-Silva (2009), por exemplo, o sistema Qualis, que deveria ponderar a qualidade das publicaçóes, acaba por reforçar a sobrevalorização da quantidade sobre a qualidade das produções no jogo de avaliação de programas e currículos de pesquisadores a serem beneficiados pelos financiamentos de pesquisa.

Este estudo limita-se a uma análise das publicações no sistema Qualis, estrato A, no ano de 2010, na área da Psicologia. Por isso, sugere-se que futuras pesquisas sejam feitas no sentido de avaliar a publicação brasileira em revistas de outros estratos do sistema Qualis ou ainda em outros períodos de tempo, ou em publicações de outros países, também avaliadas pelo sistema. Assim, uma comparação seria possível de forma a avaliar a qualidade das publicações dentro dos diferentes tipos de publicação e permitindo realizar um panorama histórico das publicações nacionais em comparação com outros contextos. Tais pesquisas são relevantes conforme buscam refletir a qualidade da publicação brasileira, se fomentam um debate acerca das peculiaridades da produção científica da Psicologia nacional, além de contribuir para uma melhoria da Ciência Psicológica no País.

\section{Referências}

Castro, L. R. (2010). Privatização, especialização e individualização: um outro mundo (acadêmico) é possível? Psicologia \& Sociedade, 22 (3), 622-627.

Clemente-Díaz, M. (1992). Psicologia social: métodos y técnicas de investigación. Madrid: Eudema. 
Coordenação de Aperfeiçoamento de Pessoal de Nível Superior. (2009). Documento de Area 2009. [on-line] Disponível em: <http://www.capes.gov. $\mathrm{br} /$ images/stories/ download/avaliacao/PSICO19jun10.pdf>.

Figueiredo, L. C. (1991). Matrizes do pensamento psicológico. Petrópolis: Vozes.

Gil, A. C. (2010). Como elaborar projetos de pesquisa. (5. ed.). São Paulo: Atlas.

Gunther, H. (2006). Pesquisa qualitativa versus pesquisa quantitativa: esta é a questão? Psicologia: Teoria e Pesquisa, 22 (2), 201-210.

Leite, T. M. (1993). Associação Nacional de Pesquisa e Pós-graduação em Psicologia (ed.). Mapeamento de pesquisa em Psicologia e áreas em descoberto. Psicologia clínica. Anais do Simpósio de Pesquisa e Intercâmbio Científico da Anpepp, 4. (pp. 65-75). Brasília: Universidade de Brasília.

Mariguela, M. (1995). Epistemologia da Psicologia. Piracicaba: Unimep.

Palmieri, M. W. \& Martins, J. B. (2008). Possibilidades e desafios da produção científica no campo da Psicologia: algumas reflexóes. Psicologia em Estudo, 13 (4), 743-752.

Prado Filho, K. \& Martins, S. A. (2007). Subjetividade como objeto da(s) Psicologia(s). Psicolologia \& Sociedade, 19 (3), 14-19.

Rocha-e-Silva, M. (2009). O novo Qualis, ou a tragédia anunciada. Clinics, 64 (1), 1-4.

Schultz D. S. \& Schultz, E. (2006). História da Psicologia Moderna. (9. ed.). São Paulo: Cengage Learning.

Serapioni, M. (2000). Métodos qualitativos e quantitativos na pesquisa social em saúde: algumas estratégias para a integração. Ciência e Saúde Coletiva, 5 (1), 187-192.

Trzesniak P. \& Koller S. H. (2009). A redação científica apresentada por editores. In: A. A. Sabadini \& M. I. Sampaio \& S. H. Koller (Org.). Publicar em Psicologia: um enfoque para a revista científica. São Paulo: Instituto de Psicologia da Universidade de São Paulo.

Trzesniak, P. (2006). As dimensões da qualidade dos periódicos científicos e sua presença em um instrumento da área da educação. Revista Brasileira de Educação, 11 (32), 346-361. 
Turato, E. R. (2005). Métodos qualitativos e quantitativos na área da saúde: definições, diferenças e seus objetos de pesquisa. Revista de Saúde Pública, 39 (3), 507-514.

Waters, L. (2006). Inimigos da esperança: publicar, perecer e o eclipse da erudição. São Paulo: Unesp. 